\title{
Label-free characterization of vitrification-induced morphology changes in single-cell embryos with full-field optical coherence tomography
}

Livia Zarnescu

Michael C. Leung

Michael Abeyta

Helge Sudkamp

Thomas Baer

Barry Behr

Audrey K. Ellerbee 


\title{
Label-free characterization of vitrification-induced morphology changes in single-cell embryos with full-field optical coherence tomography
}

\author{
Livia Zarnescu, ${ }^{a}$ Michael C. Leung, ${ }^{b}$ Michael Abeyta, ${ }^{c}$ Helge Sudkamp, ${ }^{b}$ Thomas Baer, ${ }^{d}$ Barry Behr, ${ }^{c}$ and \\ Audrey K. Ellerbee ${ }^{\mathrm{b}, \star}$ \\ aStanford University, Department of Bioengineering, 443 Via Ortega, Stanford, California 94305, United States \\ bStanford University, E.L. Ginzton Laboratory and Department of Electrical Engineering, 450 Serra Mall, Stanford, California 94305, United States \\ 'Stanford University, IVF Laboratory, Lucille Packard Children's Hospital, 900 Welch Road, Suite 350, Stanford, California 94305, United States \\ ${ }^{d}$ Stanford University, Stanford Photonics Research Center, 348 Via Pueblo Mall, Stanford, California 94305, United States
}

\begin{abstract}
Vitrification is an increasingly popular method of embryo cryopreservation that is used in assisted reproductive technology. Although vitrification has high post-thaw survival rates compared to other freezing techniques, its long-term effects on embryo development are still poorly understood. We demonstrate an application of full-field optical coherence tomography (FF-OCT) to visualize the effects of vitrification on live single-cell (2 pronuclear) mouse embryos without harmful labels. Using FF-OCT, we observed that vitrification causes a significant increase in the aggregation of structures within the embryo cytoplasm, consistent with reports in literature based on fluorescence techniques. We quantify the degree of aggregation with an objective metric, the cytoplasmic aggregation (CA) score, and observe a high degree of correlation between the CA scores of FF-OCT images of embryos and of fluorescence images of their mitochondria. Our results indicate that FF-OCT shows promise as a label-free assessment of the effects of vitrification on embryo mitochondria distribution. The CA score provides a quantitative metric to describe the degree to which embryos have been affected by vitrification and could aid clinicians in selecting embryos for transfer. @2015 Society of Photo-Optical Instrumentation Engineers (SPIE) [DOI: 10 .1117/1.JBO.20.9.096004]
\end{abstract}

Keywords: full-field optical coherence tomography; optical coherence tomography; in vitro fertilization; embryo; vitrification; cryopreservation; assisted reproductive technology; mitochondria; image processing.

Paper 150397PR received Jun. 11, 2015; accepted for publication Jul. 31, 2015; published online Sep. 3, 2015.

\section{Introduction}

Nearly 1.5 million cycles of in vitro fertilization (IVF) are performed each year worldwide, a number that has been steadily rising at a rate of 5 to $10 \%$ in recent years. ${ }^{1,2}$ A typical IVF cycle begins with ovarian hyperstimulation to produce up to 20 oocytes (eggs) at once. After fertilization and several days in culture, a morphological assessment of viability is conducted under a brightfield microscope. Typically, clinicians transfer only one to three potentially viable embryos back to the patient and freeze the rest for use in future cycles. Thus, if the patient fails to get pregnant the first time, she can forego a second stimulation treatment and instead have some of the previously frozen embryos thawed and transferred.

Because of their potential to benefit patient health and improve IVF success rates, frozen embryo transfers (FET) have risen in popularity and are now involved in $24 \%$ of IVF cycles. ${ }^{1}$ In particular, FET allows clinicians to synchronize the time of embryo transfer with the patient's optimal implantation window, ${ }^{3}$ preserve the fertility of cancer patients who are undergoing chemotherapy, ${ }^{4}$ and transfer embryos based on the results of genetic tests, which take several days to arrive. The lack of hormone injections required for FET has also resulted in a reduced incidence of ovarian hyperstimulation syndrome, which affects 1 to $2 \%$ of patients undergoing stimulation treatment and can cause serious complications. ${ }^{5,6}$

The current standard of embryo cryopreservation (embryo freezing) is by vitrification, which involves plunging embryos into liquid nitrogen to solidify them into a vitreous state. Compared to traditional slow-freezing techniques, the rapid cooling rates produced by vitrification prevent the formation of ice crystals and reduce the accumulation of toxic cryoprotectants. ${ }^{7,8}$ Vitrification is also faster, easier to perform, and has superior outcomes, such as higher rates of post-thaw survival and clinical pregnancy. ${ }^{9-11}$ When viewed under the brightfield and differential interference contrast (DIC) microscopes typically found in IVF clinics, vitrified embryos are indistinguishable from fresh embryos; however, their lower survival rates suggest that vitrification is not without deleterious effects. Indeed, studies have found that vitrification affects mitochondrial distribution and membrane potential, ${ }^{12,13}$ changes transcriptional activity, ${ }^{14}$ causes DNA fragmentation, ${ }^{15}$ and modifies cell ultrastructure. ${ }^{15,16}$ Unfortunately, tests for the aforementioned adverse effects require invasive chemical staining, fluorescence imaging, or are otherwise harmful to the embryo, making them impractical for clinical use. A few clinical studies have attempted to link vitrification, viability, and the meiotic spindle structure or embryonic secretome using label-free techniques, 
such as polarized light microscopy and mass spectrometry, but to date have yielded inconclusive results. ${ }^{17-19}$

Herein we introduce a novel use of full-field optical coherence tomography (FF-OCT) ${ }^{20-22}$ as a noninvasive, label-free method to visualize morphological changes in 2 pronuclear ( $\sin$ gle-cell) mouse embryos caused by vitrification. The onemicron axial and submicron lateral resolutions achievable with FF-OCT using ordinary light sources (halogen lamps or LEDs) and relatively low-power objectives $(20 \times)$ are comparable to that of three-dimensional (3-D) microscope systems, such as confocal and two-photon microscopes, while boasting high performance at far lower light intensities and requiring no sample preprocessing with harmful fluorescent dyes. Using a fully automated image-processing algorithm developed in our lab, we devised a new metric [the cytoplasmic aggregation (CA) score] to quantify the effects of vitrification on patterns of subcellular structures visible in embryo cytoplasm; the CA score shows high agreement with both manual assessments of morphological changes visible by FF-OCT and vitrification-induced mitochondrial clustering visible by fluorescence. Our results suggest that FF-OCT could be useful as a noninvasive, label-free approach to visualize and quantify the distribution of mitochondria and other cell structures inside embryos during the IVF process. The parameters extracted using this technique could, therefore, prove useful in assessing embryo quality after vitrification and allow clinicians to more accurately select viable embryos for transfer.

\section{Methods}

\subsection{Sample Preparation}

Fresh embryos $(n=24)$ of CD1xFVB mice were obtained from the Transgenic, Knockout, and Tumor Model Center (TKTC) at Stanford. The mice were subjected to hormone treatment with pregnant mare serum gonadotropin and human chorionic gonadotropin 72 and $24 \mathrm{~h}$ before mating, respectively. One half day after mating, the embryos were extracted from the mice and transported to our lab imaging facility for vitrification and imaging. All vitrification was performed by a trained clinical embryologist to emulate the protocol typically followed during assisted reproduction. Vitrification and thawing were performed with the Sage vitrification and warming kits (Cooper Surgical), according to the included protocols. Embryos were transported and imaged in Sage modified human tubal fluid with $10 \%$ Sage protein supplement. During imaging, a heated stage kept the media temperature around $37^{\circ} \mathrm{C}$.

\subsection{Workflow and Experiment Design}

On two separate occasions, fresh embryos were received from the TKTC $\sim 1 \mathrm{~h}$ after collection. Embryos on both days were randomly assigned to one of two groups. The embryos in the first group $(n=14)$ were imaged using FF-OCT, vitrified and thawed, and subsequently imaged with FF-OCT once more. The embryos in the second group $(n=10)$ were stained, imaged with laser scanning microscopy (LSM), imaged with FF-OCT, vitrified and thawed, imaged with FF-OCT, and then imaged with LSM. Care was taken to minimize rotation of the embryos between FF-OCT and LSM imaging sessions to ensure high fidelity and correlation of the images.

Combining the two groups, a total of 24 embryos were imaged and used in this study. Due to various challenges
Table 1 Summary of all data collected. Each table cell contains the number of embryos imaged at that step followed by the number of usable images obtained from that step. Row 3 (vitrified and thawed) contains the number of embryos vitrified followed by the number of embryos that survived the thaw and could be imaged further.

\begin{tabular}{lccc} 
& Group 1 & Group 2 & Total \\
\hline LSM previtrification & - & $10 / 9$ & $10 / 9$ \\
FF-OCT previtrification & $14 / 13$ & $10 / 9$ & $24 / 22$ \\
Vitrified and thawed & $13 / 8$ & $10 / 10$ & $23 / 18$ \\
FF-OCT postvitrification & $8 / 8$ & $10 / 10$ & $18 / 18$ \\
LSM postvitrification & - & $10 / 10$ & $10 / 10$ \\
\hline
\end{tabular}

Note: LSM, laser scanning microscopy; FF-OCT, full-field optical coherence tomography.

with embryos lysing or data corruption, the final dataset includes pre- and postvitrification FF-OCT volumes from 8 embryos in group 1, pre- and postvitrification FF-OCT volumes from 9 embryos in group 2, and pre- and postvitrification LSM datasets from 10 embryos in group 2 . Table 1 provides a detailed summary of how many embryos / datasets were acquired and maintained during the process.

\subsection{Fluorescence Imaging}

The embryos used for fluorescence imaging were incubated in PBS containing 100 nM MitoTracker Green (M7514, LifeTechnologies) according to directions from the manufacturer. Embryos were imaged using an inverted LSM780 laser scanning confocal microscope (Zeiss). The laser wavelength of $488 \mathrm{~nm}$ was chosen to coincide with the excitation wavelength of the selected dye $(490 \mathrm{~nm})$. The laser was operated at $3 \%$ of the maximum intensity to avoid damaging the embryo and photobleaching. Using a $25 \times$ LCI Plan NEO water-immersion objective (0.8 NA, Zeiss), 3-D image stacks were obtained.

\subsection{Full-Field Optical Coherence Tomography Imaging}

All embryos were imaged using the FF-OCT system shown in Fig. 1. The system is based on a Linnik interferometer and incorporates a high-power white LED (Indus Star A007-GW765-R5, LEDdynamics Inc.) focused at the back focal plane of airimmersion microscope objectives (20× Plan APO LWD, $0.42 \mathrm{NA}$, Mitutoyo). The reference arm contains a piezoelectric actuator (PZT) (Thorlabs, AE0203D08F) onto which we mounted a fragment of an absorptive neutral density filter having $\sim 4 \%$ reflectivity. An empty glass bottom Petri dish was also inserted into the reference arm for dispersion compensation. Images were acquired using a PCO Edge sCMOS camera over a small region of interest ( 860 by 800 pixels), using an integration time of $10 \mathrm{~ms}$ per frame. The incident light from the LED is a $6500 \mathrm{~K}$ color temperature, which was measured to be centered about $580 \mathrm{~nm}$. It was made to pass through a 1500-grit diffuser (Thorlabs DG10-1500-MD) and a longpass filter (Thorlabs FEL0550) that attenuates wavelengths below $550 \mathrm{~nm}$. Thus, the total intensity illuminating the embryos was measured to be $11 \mathrm{~mW} / \mathrm{cm}^{2}$ at the peak 


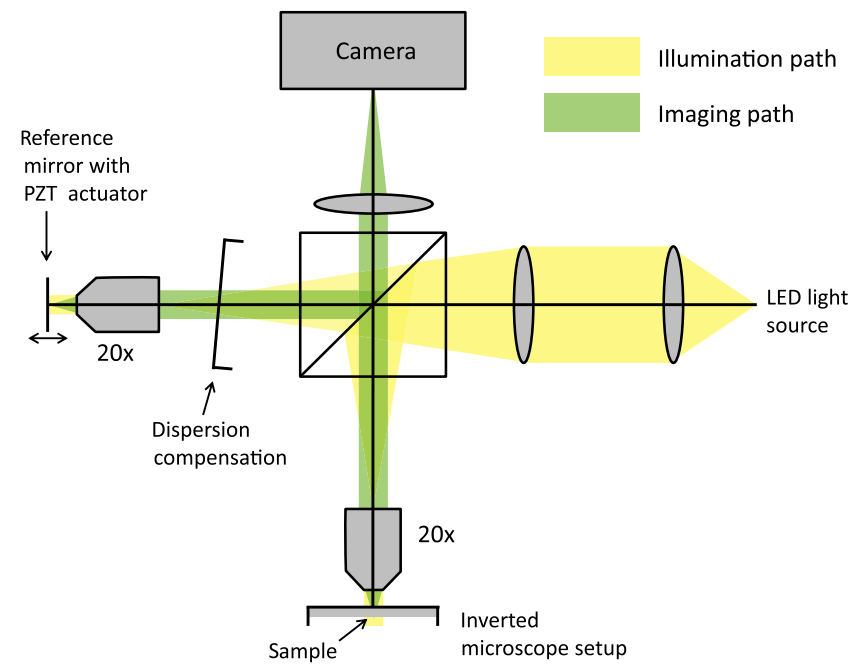

Fig. 1 Full-field optical coherence tomography (FF-OCT) system schematic. The system features an inverted microscope in the sample path to provide minimal disturbance to embryos during imaging.

wavelength of $580 \mathrm{~nm}$. The imaging sensitivity of the system was $60 \mathrm{~dB}$ but was increased by averaging. We performed accumulation of 40 to 160 images to produce a single en-face image. The number of images used was increased linearly with depth to compensate the reduced signal-to-noise ratio due to scattering and attenuation. This yielded a system sensitivity of $\sim 76 \mathrm{~dB}$ at all depths. The resolution of the system was determined to be 1.5 and $1.0 \mu \mathrm{m}$ in the axial and lateral directions, respectively. Acquisition of a 60 -stack image took, on average, $8 \mathrm{~min}$ because of the low light intensity used; however, the time can be decreased significantly by reducing the integration time and increasing the illumination intensity. A custom LabView program was written to control the position of the actuators, conduct phase stepping with the PZT, provide a trigger for the camera, and save the data in 16-bit format. The data were acquired by using a phase stepping algorithm similar to that described in Ref. 23.

\subsection{Image Processing}

The image processing steps used to isolate the cell cytoplasm for further analysis are shown in Fig. 2. Embryos underwent an initial preprocessing step to remove artifacts and ensure consistent brightness for all images in the 3-D stack. Wiener filtering and 3-D Gaussian blurring were then performed to improve the

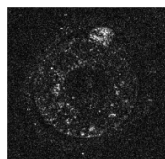

Original image

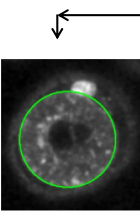

Cell outline identified

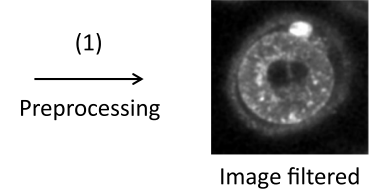

(3)

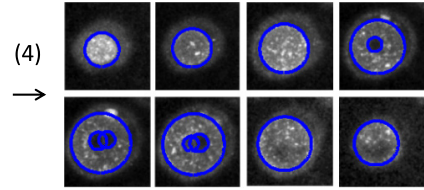

Pronuclei detected in 3-D
(2)

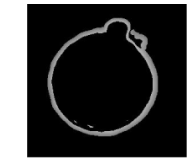

Edges detected $\downarrow$
Fig. 2 Overview of the image processing procedure performed to identify pixels inside the cell cytoplasm. signal from the embryo while suppressing noise (step 1). Edge detection and dilation were conducted on a two-dimensional projection image of the 3-D stack, and the cell cytoplasm was distinguished from the zona pellucida and background by repeated thresholding (step 2). The cell outline was identified by the application of circle Hough transform to the remaining pixels (step 3). The outlines of the cell and pronuclei in each slice were identified via repetition of steps 2 and 3 on each slice in the 3-D stack of images (step 4). Finally, a 3-D binary mask was created to identify the embryo cytoplasm (step 5).

\section{Results}

\subsection{Full-Field Optical Coherence Tomography Reveals Visible Changes in Embryos after Vitrification}

We imaged one-cell mouse embryos before and after vitrification using both FF-OCT and fluorescence microscopy; as described previously, embryos in group 2 were stained to enable visualization of mitochondria. Consistent with prior literature, ${ }^{12,24}$ we found qualitative changes in the distribution of the intensity signal from mitochondria before and after vitrification in the fluorescence images, which also appeared to correlate with the distribution of high-intensity structures we observed in the FF-OCT images (Fig. 3). In both imaging modalities, the intensity signal appears fairly evenly distributed in fresh embryos before vitrification. After vitrification, mitochondria seem to aggregate in clusters close to the center of the embryo; similarly, there is a clustering of bright structures seen in the FF-OCT images after vitrification. The two imaging modalities appear quite similar, which implies that a significant component of the FF-OCT signal may come from light scattering and reflection caused by mitochondria.

\subsection{Changes in Embryo Cytoplasm Can Be Quantified with Our Automated Algorithm}

In order to more precisely quantify the changes we saw in embryos due to vitrification, we developed an image processing algorithm to extract parameters from the FF-OCT images of

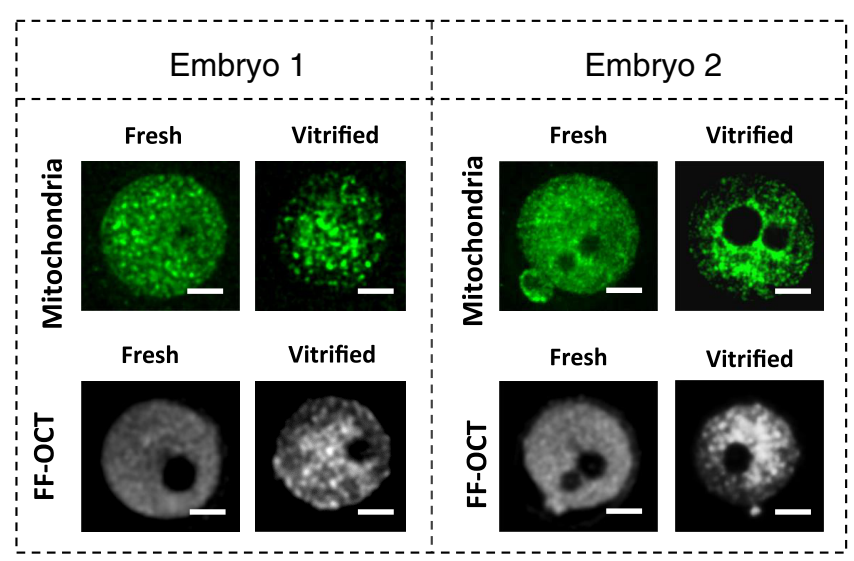

Fig. 3 Vitrification changes the distribution of mitochondria in embryos, as seen in images of fluorescently stained mitochondria. Similar changes are also visible with FF-OCT. Two example embryos from our dataset are shown. For each embryo, all four images are from a single slice of the three-dimensional volume and are taken from the same embryo at the $z$-location with the maximum radius. Scale bar $=20 \mu \mathrm{m}$. 
fresh $(n=22)$ and vitrified $(n=18)$ embryos. We thus devised a mechanism to score embryos based on our general observations of the clustering of cellular contents. Extraction of the parameters to quantify such $\mathrm{CA}$ in the embryo is described below.

First, the intensity profile within the embryo cytoplasm was calculated going radially outward for all slices bordering the pronuclei in three dimensions. To maximize the signal associated with aggregation near the pronuclei and cell membranes, only slices contained within $5 \mu \mathrm{m}$ of the centroid of the pronuclei were considered. For each slice, the cytoplasm of the embryo was split into 20 concentric disks of equal radius, and the average intensity of all cytoplasmic pixels in each disk was calculated [Fig. 4(a)]. Pixels inside the pronuclei and outside the cell boundary were excluded from the average. The average intensity from each disk was plotted as a function of disk radius, yielding a radial intensity profile for each embryo.

The radial intensity profiles for all slices within $5 \mu \mathrm{m}$ of the pronuclei were averaged to generate a final radial intensity profile for each embryo. Figure 4(b) shows radial intensity profiles of embryos having differing cytoplasmic distributions, with representative images of embryos having cytoplasmic distributions that were homogeneous or aggregated (classified manually) shown alongside for reference. From these distributions, it is evident that the embryos with manually classified homogeneous distributions have intensity profiles that are generally flat or increase slightly toward the outside of the cell. The embryos with manually classified aggregated distributions show intensity profiles that are high in the inner $50 \%$ of the cell and then appear to drop off toward the outside.

We then calculated various features based on the radial intensity profiles that were linear combinations of the elements of the radial intensity profile vector (i.e., sums and differences of various points along the intensity profile) and calculated the accuracy with which these features could reproduce our manually classified cytoplasm distributions. We ultimately found that a single feature was sufficient to automatically classify the embryos and that our automatic classification showed good agreement with our manual classification. We call this feature the CA score, and it is calculated as follows:

CA score $=r_{50 \%}-r_{75 \%}$, where $r$ is the radial intensity profile vector (which has 20 total elements representing the concentric disks into which we segmented the cell cytoplasm), and the subscripts of $r$ represent the radius (as a percentage of the total vector length) at which to evaluate its value [see Fig. 4(a)]. For example, $r_{50 \%}$ corresponds to the 10th element of the $r$ vector and $r_{75 \%}$ corresponds to the 15th element of the $r$ vector. Using this metric, an embryo with a high amount of aggregation should have a high (positive) CA score, and an embryo with a homogeneous cytoplasm should have a low (negative) CA score. Hence, the embryo with homogenous cytoplasm, depicted in Fig. 4(b) (top), possesses a CA of nearly zero, as the intensity is constant throughout the cytoplasm. On the other hand, the embryo with the aggregated cytoplasm, depicted in Fig. 4(b) (bottom), exhibits a positive CA score, resulting from the ring located at $50 \%$ of the maximum radius being significantly brighter than that at $75 \%$ of the maximum radius.

The CA scores of embryos with manually classified aggregated and homogeneous cytoplasm distributions exhibited only a small degree of overlap. Figure 5(a) shows a smoothed kernel density plot of the CA scores. In Fig. 5(b), we show a receiver operating characteristic (ROC) curve that evaluates the performance of the CA score as a classifier. The area under the ROC curve is 0.94 , which is indicative of a highly accurate classifier. It should be noted that the ground truth classification (i.e., manual classification as homogeneous or aggregated) is itself subject to human error. In particular, upon visual inspection, some embryos appeared to be slightly aggregated or in-between the two patterns. Because our automatic classifier lacks the bias and subjectivity of a human, its accuracy may be understated.

\subsection{Vitrification Causes an Increase in Embryo Cytoplasmic Aggregation}

We next sought to determine whether vitrification induced significant changes in the distribution of the FF-OCT signal within embryo cytoplasm distribution. Using the CA scores we had previously calculated for our fresh $(n=22)$ and vitrified $(n=18)$ embryos, we found that vitrification caused significant changes in embryo cytoplasm morphology $(p \ll 0.001)$ (Fig. 6). After vitrification, 94\% of the embryos for which we had both pre- and postvitrification images exhibited an (a)

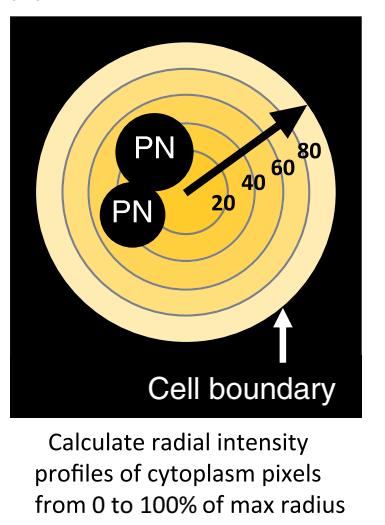

(b)

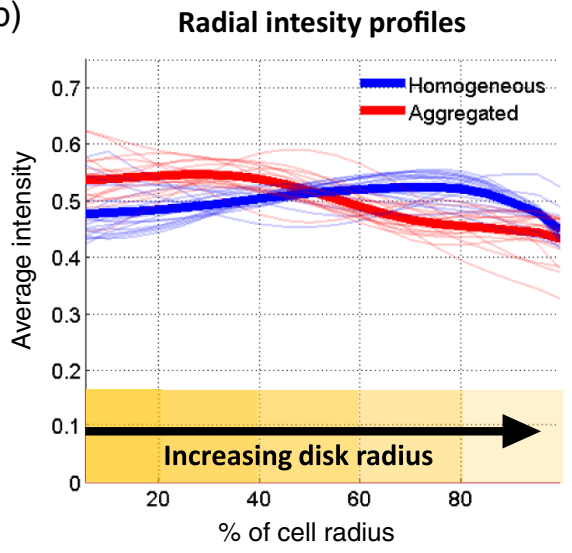

Homogeneous cytoplasm

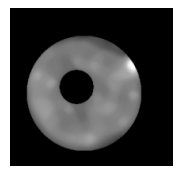

Aggregated cytoplasm

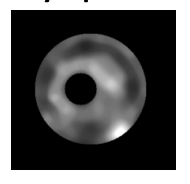

Fig. 4 Method to extract a cytoplasmic aggregation (CA) score. (a) Schematic of the radial segmentation of embryos for extracting the radial intensity profile. (b) Qualitative comparison of radial intensity profile shapes of embryos with high and low aggregation, as scored manually. Images of typical embryos with aggregated cytoplasm and a homogeneous cytoplasm are shown alongside the plot for reference. 
(a) Automatic morphology classification

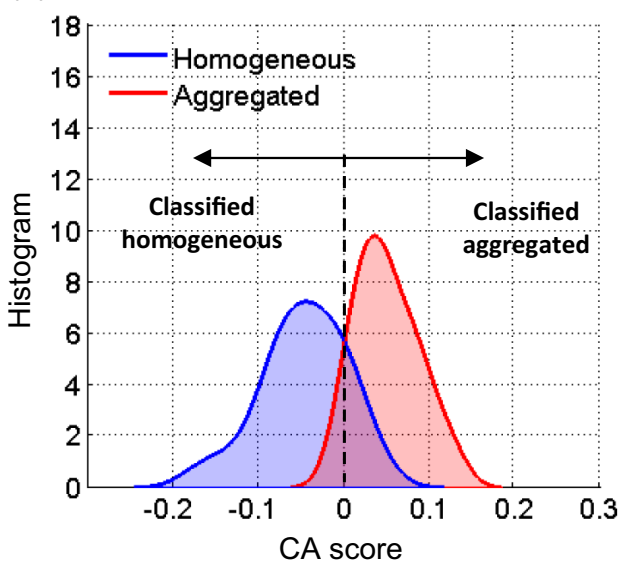

(b) ROC curve for morphology classification

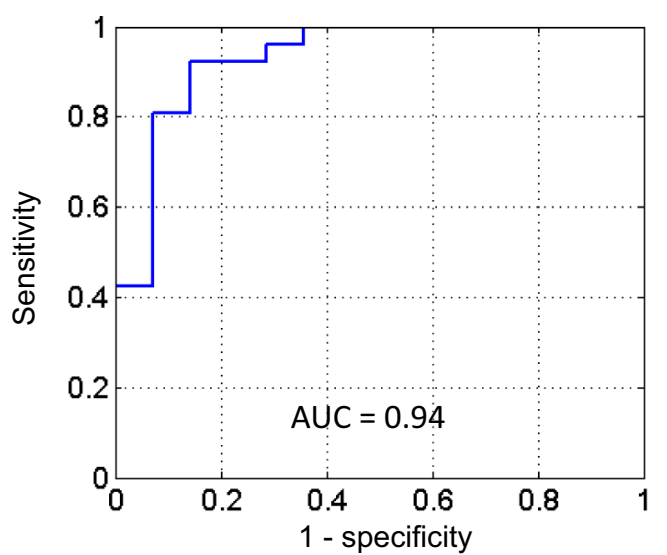

Fig. 5 (a) The CA score extracted from the radial intensity profile can accurately reproduce manual classification of embryo cytoplasm distributions. (b) The area under the ROC curve is 0.94 when using the CA score to distinguish embryos by clumping.

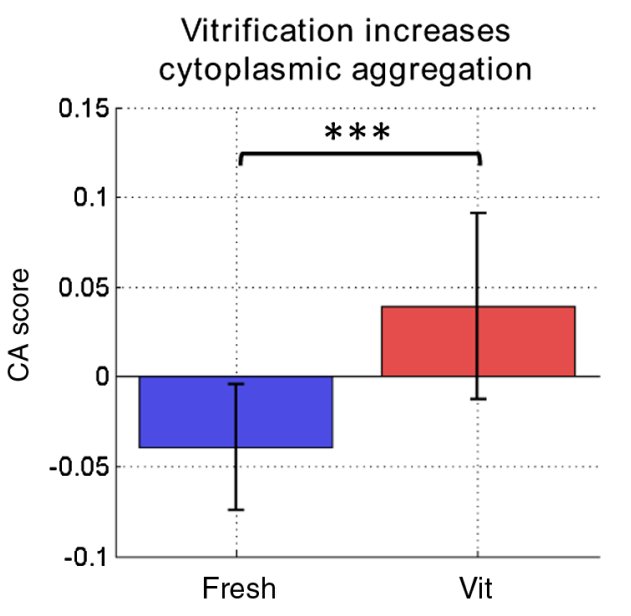

Fig. 6 Cytoplasm aggregation quantified from FF-OCT images increases postvitrification. The CA score between fresh $(n=22)$ and vitrified $(n=18)$ embryos shows a statistically significant difference $(p<1 \mathrm{e}-6)$. increase in the CA score. On average, the change in the CA score after vitrification was 0.084 with a standard deviation of 0.057 .

We also calculated a CA score for the fluorescence images of mitochondria to confirm that vitrification causes aggregation of the intensity signal for this imaging modality. We applied a similar processing method to the fluorescence images to extract radial intensity profiles and then calculated the CA score of embryos before $(n=10)$ and after $(n=10)$ vitrification. It is important to note that in this case, the CA score directly correlates with the distribution of mitochondria. As shown in Fig. 7(a), the radial intensity profiles of the fluorescence images appear slightly different from those of the FF-OCT images: the radial intensity profiles of the fluorescence images are dim near the outer edge of the cell because they lack the bright signal from the cell membrane that is seen in many FF-OCT images.

Otherwise, the radial intensity profiles of the fluorescence images are similar to those of the FF-OCT images in that the profiles of vitrified embryos experience a drop-off in signal closer to the center of the cell compared to fresh embryos. (a)

Vitrification changes distribution of mitochondria

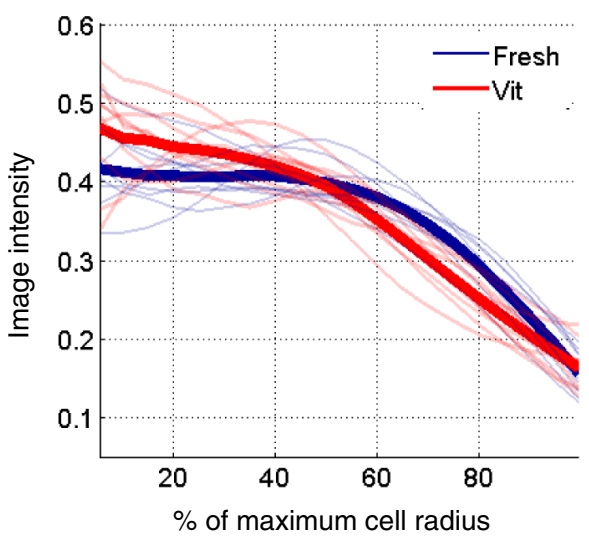

(b)

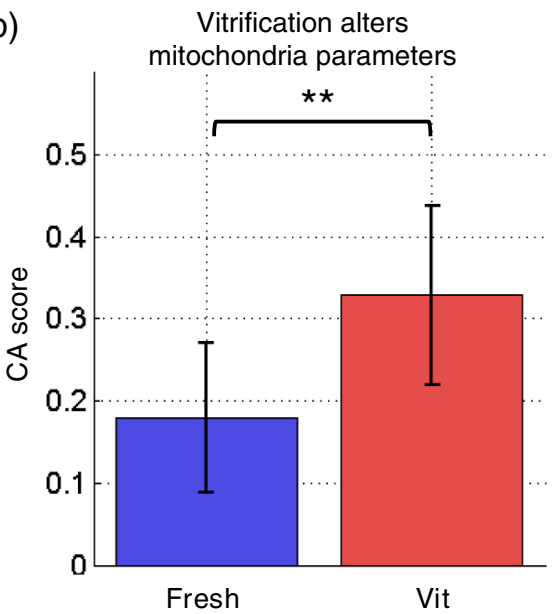

Fig. 7 The radial intensity profile extracted from fluorescent images of mitochondria quantifies the increased aggregation of mitochondria in the center of the cell postvitrification. (a) While these images do not possess a bright outline at the edges seen in FF-OCT, the radial profile shows a steeper decline away from the center in vitrified embryos compared to previtrification. (b) The mitochondria show significantly more aggregation after vitrification based on the CA score $(p=0.006)$. 


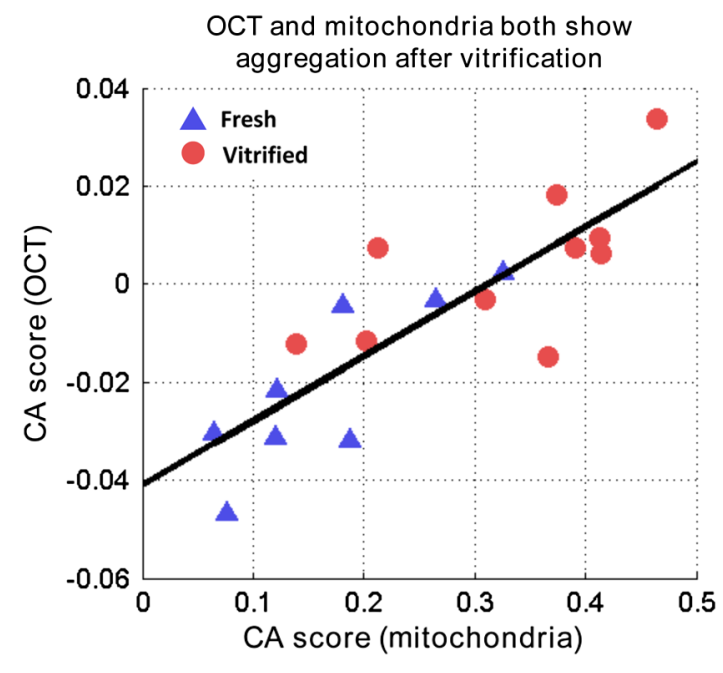

Fig. 8 Embryos that show aggregation under FF-OCT also show aggregation of stained mitochondria ( $n=8$ for previt, $n=10$ for postvit). The correlation between the two imaging modalities ( $r$-square $=$ $0.42, p=0.002$ ) suggests that the FF-OCT signal partly originates from mitochondria.

When quantifying the differences in the radial intensity profiles with the $\mathrm{CA}$ score, it can be seen that vitrified embryos again have a higher CA score than fresh embryos. Therefore, the CA score accurately captured a significant $(p=0.005)$ increase in aggregation of mitochondria in vitrified embryos [Fig. 7(b)].

\subsection{Full-Field Optical Coherence Tomography May Be Used as a Label-Free Measure of Mitochondria Distribution}

Because we observed that the fluorescence and FF-OCT images appeared qualitatively very similar, we sought to determine the extent to which the FF-OCT signal likely originated from the mitochondria and, therefore, whether FF-OCT could indirectly provide insight into the effects of vitrification on embryo mitochondria. We compared the CA scores of embryos that were imaged with both fluorescence and FF-OCT $(n=10)$ and found a visible relationship between the CA scores from the two imaging modalities (Fig. 8). Our results suggest that aggregation in FF-OCT statistically correlates with mitochondrial aggregation viewed with fluorescence with high significance $(p=0.002, r$-squared $=0.42)$. Hence, we conclude that, aside from the bright signal from the cell membrane, a significant component of the FF-OCT signal must originate from the distribution of mitochondria.

\section{Discussion}

In this study, we replicated former results showing that vitrification induces clustering of mitochondria in embryos and demonstrated that the FF-OCT signal from such embryos appears very similar to the fluorescence signal from the mitochondria of those embryos. Specifically, if an embryo contains a highly aggregated intensity signal within its cytoplasm under FF-OCT, then its mitochondria will most likely show this same pattern under fluorescence imaging. The high sensitivity of embryos to light exposure and to injected chemical dyes usually precludes the use of fluorescence imaging as a diagnostic or screening tool in the IVF clinic. ${ }^{25,26}$ Even in a research setting, it is still challenging to stain and image live embryos and observe their development afterward. Because FF-OCT imaging is label-free and requires 6 to 10 orders of magnitude lower light exposure than imaging techniques such as confocal and two-photon microscopy, ${ }^{27,28}$ our results suggest that it could serve as an indirect measure of mitochondrial distribution in live embryos that may be correlated with the effects of vitrification. Notably, the results were consistent for both group 1 and group 2 embryos, suggesting that the staining procedure was not a confounding factor for the FF-OCT imaging results.

In addition to demonstrating qualitative differences between fresh and vitrified embryos under FF-OCT imaging, we also developed a quantitative measure of these differences that we called the CA score. Manual classification is binary and also highly subjective, so we believe that an automated approach can offer finer resolution of the degree to which each embryo is affected by vitrification. Further work in quantifying the effects of vitrification could supplement the CA score with features describing the texture of the cytoplasm after vitrification. The radial intensity profiles could also be modified to describe the signal intensity from the center of each pronucleus toward the cell membrane: we observed such an aggregation of structures around pronuclei rather than just in the center of each embryo but did not investigate it extensively in this work.

Other label-free imaging modalities used in the IVF clinic, such as brightfield and DIC, are commonly used to record simple morphological parameters such as the number of cells, degree of fragmentation, pronucleus formation, and general cytoplasm appearance. However, neither of these techniques is capable of observing the distribution of organelles within the embryo cytoplasm; hence, they may not provide embryologists with complete information about the internal state of an embryo after vitrification. Our work suggests that the use of FF-OCT to observe differences in mitochondrial distribution within a heterogeneous group of embryos, or to determine whether some embryos have been more affected by vitrification and thawing than others, may have high clinical value, and the label-free nature of FF-OCT makes it easy to translate this technology for clinical use.

To determine whether exposure to light during FF-OCT imaging may compromise embryo viability, we conducted a separate experiment where we cultured embryos for five to six days after imaging (data not shown). The blastocyst formation rate was similar to that typical of vitrified mouse embryos that have not been imaged with FF-OCT, which suggests that FFOCT itself is likely not harmful to the embryos. To minimize potential harm to embryos during imaging, we significantly lowered the light intensity during imaging while increasing camera exposure time, and we also added a high-pass filter at $550 \mathrm{~nm}$ into our illumination path to avoid exposing embryos to blue light wavelengths that could lower viability. While these modifications may have reduced the impact of imaging on the embryos, they resulted in a significantly increased stack acquisition time and lowered axial resolution. Further work will have to be done in both mouse and human embryos to thoroughly understand the relationship between FF-OCT imaging parameters and embryo development rates in culture. In addition, our FF-OCT system requires very precise alignment to achieve good image quality and is very sensitive to vibrations. More work is required to increase the robustness of this system before translating it into the IVF clinic for routine use.

Previous studies in the literature have shown that vitrification may alter the distribution of mitochondria, which can then affect embryo viability due to asymmetric distribution of ATP and 
other products. ${ }^{12,24,29}$ While it is well understood that changes in the arrangement of intracellular structures may affect embryo metabolism and viability, some studies $^{30}$ have also shown that vitrification is not the only potential source of mitochondrial aggregation. One study found that CA can arise near the pronuclei immediately prior to cell division $;{ }^{24}$ hence, some of the patterns we observed may be associated with cell cycle timing rather than the effects of vitrification. We believe that cell cycle timing had a minimal confounding effect on our data because the timing between our pre- and postvitrification imaging sessions was only $150 \mathrm{~min}$, which is much shorter than a full cell cycle $(\sim 20 \mathrm{~h})$. To further control for the effect of cell cycle timing, we also imaged a group of four embryos every 5 min for $24 \mathrm{~h}$ to observe one cell division, and we did not observe increased aggregation until 5 to $10 \mathrm{~min}$ before cell division (data not shown). Because our embryos did not divide for several hours after FF-OCT imaging, we do not think cell cycle timing was a confounding factor in our experiments. Moreover, because the FF-OCT signal we observed is highly correlated to the fluorescence signal from mitochondria, it is highly likely that the FF-OCT signal that we associated with aggregated structures was a strong, indirect measure of mitochondrial distribution. Follow-up work to this study could determine if the changes we see in the FF-OCT signal after vitrification are also predictive of outcome measures such as embryo survival in culture.

The work in this study represents an important step toward validating FF-OCT as a tool to evaluate embryo morphology and toward improving noninvasive prediction of embryo viability. As frozen embryo transfer becomes more common during IVF and more women elect to freeze their eggs, it will be important to understand the effects of the freezing and thawing process on embryo viability. The process of vitrification is consistently improving as it overtakes slow freezing in popularity, so FFOCT could provide important insight into the effects of different vitrification protocols and equipment. Furthermore, it is still difficult to assess how vitrification affects embryos at the molecular level. Most clinicians look at survival directly after thawing to evaluate whether an embryo has been damaged by vitrification; however, some changes in the embryo structure or biochemistry could persist after thawing that are not detectable by brightfield or DIC. We believe FF-OCT could help address this problem by providing clinicians with better information about embryo cytoplasmic organization after thawing, and in the future, it could even be applied to visualize human embryo development in 3-D and find new parameters predictive of developmental potential.

\section{Acknowledgments}

L.Z. was funded by an NSF Graduate Fellowship and the Stanford Graduate Fellowship. This work and M.C.L. were funded by NSF CBET-1351981. Preliminary studies were funded by a CBIS seed grant from Stanford. The authors would like to acknowledge the Stanford Transgenic, Knockout, and Tumor Model Center for providing the embryos and the Shriram Center Cell Sciences Imaging Facility at Stanford for providing access to the laser scanning microscopy fluorescence microscope.

\section{References}

1. A. P. Ferraretti et al., "Assisted reproductive technology in Europe, 2009: results generated from European registers by ESHRE," Hum. Reprod. 28(9), 2318-2331 (2013).

2. Centers for Disease Control and Prevention, American Society for Reproductive Medicine, Society for Assisted Reproductive Technology,
"2011 assisted reproductive technology fertility clinic success rates report," US Dept. of Health and Human Services, Atlanta, Georgia (2013).

3. M. Roque et al., "Fresh embryo transfer versus frozen embryo transfer in in vitro fertilization cycles: a systematic review and meta-analysis," Fertil. Steril. 99(1), 156-162 (2013).

4. G. Bedoschi and K. Oktay, "Current approach to fertility preservation by embryo cryopreservation," Fertil. Steril. 99(6), 1496-1502 (2013).

5. P. S. Gera et al., "Ovarian hyperstimulation syndrome: steps to maximize success and minimize effect for assisted reproductive outcome," Fertil. Steril. 94(1), 173-178 (2010).

6. P. Humaidan, J. Quartarolo, and E. G. Papanikolaou, "Preventing ovarian hyperstimulation syndrome: guidance for the clinician," Fertil. Steril. 94(2), 389-400 (2010).

7. J. Liebermann et al., "Potential importance of vitrification in reproductive medicine," Biol. Reprod. 67, 1671-1680 (2002).

8. K. E. Loutradi et al., "Cryopreservation of human embryos by vitrification or slow freezing: a systematic review and meta-analysis," Fertil. Steril. 90(1), 186-193 (2008).

9. L. L. Kuleshova and A. Lopata, "Vitrification can be more favorable than slow cooling," Fertil. Steril. 78(3), 449-454 (2002).

10. E. Stehlik et al., "Vitrification demonstrates significant improvement versus slow freezing of human blastocysts," Reprod. Biomed. Online 11(1), 53-57 (2005).

11. B. Balaban et al., "A randomized controlled study of human day 3 embryo cryopreservation by slow freezing or vitrification: vitrification is associated with higher survival, metabolism and blastocyst formation," Hum. Reprod. 23(9), 1976-1982 (2008).

12. L. Ren et al., "Effects of mechanical delipation in porcine oocytes on mitochondrial distribution, ROS activity and viability," Cryo Lett. 36(1), 30-36 (2015).

13. X.-M. Zhao et al., "Effect of vitrification on mitochondrial distribution and membrane potential in mouse two pronuclear (2-PN) embryos," Mol. Reprod. Dev. 76(11), 1056-1063 (2009).

14. A. Dhali et al., "Gene expression and development of mouse zygotes following droplet vitrification," Theriogenology 68, 1292-1298 (2007).

15. D. Fabian et al., "Ultrastructure and cell death of in vivo derived and vitrified porcine blastocysts," Mol. Reprod. Dev. 70(2), 155-165 (2005).

16. T. Fair et al., "Ultrastructure of bovine blastocysts following cryopreservation: effect of method of blastocyst production," Mol. Reprod. Dev. 58(2), 186-195 (2001).

17. G. Leoni et al., "Resumption of metabolic activity of vitrified/warmed ovine embryos," Mol. Reprod. Dev. 64(2), 207-213 (2003).

18. F. Dominguez et al., "Effect of vitrification on human oocytes: a metabolic profiling study," Fertil. Steril. 99(2), 565-572 (2013).

19. C. K. Chen et al., "Evaluation of meiotic spindles in thawed oocytes after vitrification using polarized light microscopy," Fertil. Steril. 82(3), 666-672 (2004).

20. A. Dubois et al., "High-resolution full-field optical coherence tomography with a Linnik microscope," Appl. Opt. 41(4), 805-812 (2002).

21. E. Beaurepaire et al., "Full-field optical coherence microscopy," Opt. Lett. 23(4), 244-246 (1998).

22. A. Dubois et al., "Ultrahigh-resolution full-field optical coherence tomography," Appl. Opt. 43, 2874-2883 (2004).

23. K. Creath, "Phase-measurement interferometry techniques," in Progress in Optics, E. Wolf, Ed., Vol. 26, pp. 349-393, North Holland, Amsterdam, Netherlands (1988).

24. B. D. Bavister and J. M. Squirrell, "Mitochondrial distribution and function in oocytes and early embryos," Hum. Reprod. 15(Suppl 2), 189-198 (2000).

25. R. M. Schultz, "Of light and mouse embryos: less is more," Proc. Natl. Acad. Sci. 104(36), 14547-14548 (2007).

26. Y. Hirao and R. Yanagimachi, "Detrimental effect of visible light on meiosis of mammalian eggs in vitro," J. Exp. Zool. 206, 365-369 (1978).

27. K. Konig, "Multiphoton microscopy in life sciences," J. Microsc. 200, 83-104 (2000).

28. J. B. Pawley and B. R. Masters, "Handbook of biological confocal microscopy, third edition," J. Biomed. Opt. 13(2), 029902 (2008).

29. S. Nagai et al., "Correlation of abnormal mitochondrial distribution in mouse oocytes with reduced developmental competence," Tohoku J. Exp. Med. 210(2), 137-144 (2006).

30. V. Noto et al., "Mitochondrial distribution after fast embryo freezing," Hum. Reprod. 8(12), 2115-2118 (1993). 
Livia Zarnescu is a $\mathrm{PhD}$ candidate in bioengineering at Stanford University and is working on developing new biomarkers of embryo developmental potential. She received an MS in bioengineering from Stanford University in 2012 and a BS in optical engineering and mathematics from the University of Arizona, Tucson, Arizona in 2010. Her research interests include cell mechanics, microscopy, machine learning, and bioinformatics with applications to developmental biology.

Michael C. Leung is currently a PhD student in electrical engineering, and a MSc student in mechanical engineering at Stanford University. Prior to Stanford, he completed his Bachelor of Applied Science in engineering at the University of Waterloo, Ontario, Canada in 2014. His research interests are in medical imaging and medical devices, where he hopes to develop new MEMS and microfluidic devices for clinical applications.

Thomas Baer is the executive director of the Stanford Photonics Research Center, a consulting professor in the Applied Physics Department and an associate member of the Stem Cell Institute at Stanford University. His current scientific research is focused on developing imaging and biochemical analysis technology for exploring the molecular basis of human developmental biology and neuroscience. He received a BA in physics from Lawrence University in 1974, and a $\mathrm{PhD}$ in atomic physics from the University of Chicago in 1979.

Barry Behr is a nationally and internationally renowned clinical and scientific leader in the research and advances of human reproduction. $\mathrm{He}$ is the first non-MD president of the Pacific Coast Reproductive Society. $\mathrm{He}$ is the director of the IVF/ART laboratory and co-director of the REI-IVF program both at Stanford University and is the director of the Renew Biobank Institute for Stem Cell Biology and Regenerative Medicine and is in the forefront of clinical and scientific advances in reproduction, nationally and internationally.

Audrey K. Ellerbee holds a BSE in electrical engineering from Princeton University and a PhD in biomedical engineering from Duke University. She completed her postdoctoral training at Harvard University. Audrey joined Stanford University and the Ginzton Laboratory in September 2010, and is a member of the Optical Society of America and SPIE. Her research interests span microscopy, optical coherence tomography, optofluidics, and low-cost diagnostics.

Biographies for the other authors are not available. 